\title{
EFECTO DE LA MACA ROJA (Lepidium meyenii) SOBRE LOS NIVELES DE IFN- $\gamma$ EN RATAS OVARIECTOMIZADAS
}

\author{
Johanna Leiva-Revilla ${ }^{1,2}$, Félix Guerra-Castañon²,4, Paola Olcese-Mori'1,2, Iván Lozada ${ }^{5,6}$, \\ Julio Rubio ${ }^{1,2,3}$, Carla Gonzales ${ }^{1,2,3}$, Gustavo F. Gonzales $1,2,3$
}

\begin{abstract}
RESUMEN
Objetivos. Comparar el efecto de diferentes dosis de maca roja sobre los niveles de interferón gamma (IFN-ץ) en ratas ovariectomizadas (OVX). Materiales y métodos. Ratas hembras adultas fueron divididas al azar en los siguientes seis grupos: Grupo 1: ratas pesudo-ovariectomizadas (PO); Grupo 2: ratas OVX; Grupo 3: ratas OVX tratadas con $4 \mathrm{ug} / \mathrm{kg}$ de estradiol, y Grupo 4, 5 y 6: ratas OVX tratadas con extractos de maca con 2,15, 4,3 y 8,6 mg polifenoles/kilogramo de peso corporal, respectivamente. Resultados. Las ratas OVX mostraron niveles bajos de IFN-y en comparación con las ratas $\mathrm{PO}$. El estradiol y la maca roja revirtieron el efecto de la ovariectomía sobre los niveles de IFN-y. La maca roja presenta un incremento dosis-respuesta de los niveles de IFN-y $(r=0,57, p<0,05)$. Conclusiones. La administración de la maca roja incrementa los niveles de IFN-y en ratas ovariectomizadas.
\end{abstract}

Palabras clave: Maca; Interferón gamma; Ovariectomía; Estradiol (fuente: DeCS BIREME).

\section{EFFECT OF RED MACA (Lepidium meyenii) ON IFN- $\gamma$ LEVELS IN OVARIECTOMIZED RATS}

\begin{abstract}
Objectives. Compare the effect of different doses of red maca on gamma interferon (IFN- $\gamma$ ) levels in ovariectomized rats (OVX). Materials and methods. Adult female rats were randomly divided into the following six groups: Group 1: pseudo-ovariectomized rats (PO); Group 2: OVX rats; Group 3: OVX rats treated with $4 \mathrm{ug} / \mathrm{kg}$ estradiol; and Group 4, 5 and 6: OVX rats treated with red maca extracts with $2.15,4.3$ and $8.6 \mathrm{mg}$ polyphenols/body weight kilogram, respectively. Results. OVX rats showed low levels of IFN- $\gamma$ compared to PO rats. Estradiol and red maca reversed the effect of ovariectomy on the IFN- $\gamma$ levels. A positive dose-response effect of red maca on IFN- $\gamma$ levels was shown $(r=0.57, p<0.05)$ Conclusions. Red maca administration increases levels of IFN- $\gamma$ in ovariectomized rats.
\end{abstract}

Key words: Maca; Interferon-gamma; Ovariectomy; Estradiol (source: MeSH, NLM).

\section{INTRODUCCIÓN}

La menopausia incrementa el riesgo de sufrir condiciones crónicas como enfermedad coronaria cardiaca, síndrome metabólico, osteoporosis, desordenes cognitivos y de estados de ánimo (1), debido a una disminución de la producción endógena de estrógenos por parte de los ovarios (2). Por ejemplo, una reducción en los niveles de estrógenos circulantes está asociada a la disminución de los niveles de interferón gamma (IFN-y) (3) el cual, a su vez, podría incrementar el riesgo de osteoporosis en mujeres de edad (4). De hecho, en mujeres con osteoporosis se encuentran bajos niveles de IFN- ${ }^{(5)}$

La terapia hormonal de reemplazo (THR) ha demostrado regular el sistema inmune en mujeres posmenopaúsicas ${ }^{(6-8)}$. De hecho, la THR muestra un incremento en los niveles de IFN- $\gamma$ en mujeres con menopausia natural y quirúrgica ${ }^{(3)}$. Así mismo, la THR reduce las fracturas relacionadas a la osteoporosis en mujeres con síntomas de la menopausia (9) debido a su capacidad para regular los niveles de IFN-y en mujeres con deficiencia de estrógenos ${ }^{(5,10)}$. Sin embargo, la THR

\footnotetext{
Laboratorio de Endocrinología y Reproducción, Facultad de Ciencias y Filosofía. Universidad Peruana Cayetano Heredia. Lima-Perú.

Departamento de Ciencias Biológicas y Fisiológicas, Facultad de Ciencias y Filosofía, Universidad Peruana Cayetano Heredia. Lima-Perú.

Instituto de Investigaciones de la Altura, Universidad Peruana Cayetano Heredia. Lima-Perú.

Facultad de Medicina, Universidad Peruana Cayetano Heredia. Lima-Perú.

Laboratorio de Inmunología, Facultad de Ciencias y Filosofía, Universidad Peruana Cayetano Heredia. Lima-Perú.

6 Departamento de Microbiología, Facultad de Ciencias y Filosofía, Universidad Peruana Cayetano Heredia. Lima-Perú.

Recibido: : 24-06-14 Aprobado: 05-11-14
} 
tiene varios efectos secundarios, como cáncer de mama, fallas vasculares a nivel cardiaco y cerebral y coágulos sanguíneos (11). Por estas razones, son necesarios tratamientos alternativos $\sin$ efectos secundarios adversos.

La maca (Lepidium meyenii) es una planta peruana perteneciente a la familia de las Brassicaceaes. Estudios previos han demostrado las propiedades tradicionales de los hipocótilos de maca, relacionadas con la mejora de la fertilidad, sobre la producción espermática ${ }^{(12-14)}$, conducta sexual ${ }^{(15,16)}$, y fertilidad femenina ${ }^{(17)}$. La variedad roja de maca ha demostrado reducir el peso prostático en ratas macho con hiperplasia prostática benigna inducida por enantato de testosterona ${ }^{18,19)}$ y tener un efecto protector sobre la arquitectura ósea en ratas ovariectomizadas (20), un modelo animal que imita los síntomas de la menopausia ${ }^{(21)}$. Aunque estudios in vitro han demostrado que el tratamiento con maca induce la tasa de producción de IFN-Y ${ }^{(22)}$, no se han realizado estudios in vivo con diferentes dosis de maca roja.

A partir del hecho de que la maca roja pueda reducir experimentalmente la hiperplasia prostática benigna y la osteoporosis, el presente estudio tiene como finalidad comparar el efecto de diferentes dosis de maca roja sobre los niveles de IFN-Y ratas ovariectomizadas (OVX).

\section{MATERIALES Y MÉTODOS}

\section{ANIMALES}

Se obtuvieron treinta seis ratas hembra de la cepa Holtzman (3 meses de edad) del Bioterio de la Universidad Peruana Cayetano Heredia (Lima, Perú). Las ratas (peso corporal inicial: 214,75 $\pm 1,49 \mathrm{~g}$ ) fueron colocadas en jaulas en seis ratas por jaula y mantenidas bajo condiciones controladas a $22{ }^{\circ} \mathrm{C}$ y con un ciclo de luz/oscuridad de 12:12 h dentro del Bioterio de la Universidad Peruana Cayetano Heredia. Todas las ratas recibieron comida y agua ad libitum.

\section{OVARIECTOMÍA}

Para el procedimiento, las ratas fueron anestesiadas con $40 \mathrm{mg} / \mathrm{kg}$ de pentobarbital vía intraperitoneal (i.p.). La ovariectomía bilateral fue realizada a nivel dorsolateral. Luego, los ovarios y el tejido adiposo adyacente fueron removidos y finalmente, se realizó la sutura de músculo y piel. Procesos quirúrgicos similares fueron llevados a cabo para obtener un grupo de ratas pseudoovariectomizadas (PO) donde se realizó el mismo procedimiento, pero sin remover los ovarios.
Todos los experimentos fueron realizados una semana después de la cirugía ${ }^{(23)}$.

\section{PREPARACIÓNDELEXTRACTOHIDROALCOHÓLICO DE MACA ROJA}

Los hipocótilos secos de maca roja fueron obtenidos de la ciudad de Carhuamayo en Junín, a 4000 metros de altitud, en los Andes Centrales del Perú donde se cultiva tradicionalmente ${ }^{(24)}$. en el Departamento de Ciencias Farmacéuticas de la Universidad Peruana Cayetano Heredia se autentificó la identidad de la planta. Además, una muestra de la planta (IFV 1885) fue almacenada en el Departamento.

El extracto hidroalcohólico estandarizado de los hipocótilos de maca roja fue preparado con etanol acuoso $(60 \% \mathrm{v} / \mathrm{v})$ por filtración a temperatura ambiente por $24 \mathrm{~h}$ y concentrado a presiones bajas a peso constante. Un gramo de hipocótilos secos de maca roja produce $0,22 \mathrm{~g}$ de extracto hidroalcohólico de maca roja. Este extracto fue diluido en agua destilada para obtener diferentes concentraciones en $1 \mathrm{~mL}$. Las soluciones fueron colocadas en viales y mantenidas refrigeradas a $4{ }^{\circ} \mathrm{C}$ hasta su uso ${ }^{(23)}$. El extracto hidroalcohólico de maca roja fue estandarizado según su contenido de polifenoles utilizando la reacción de Follin-Ciocalteu descrita en estudios previos ${ }^{(25)}$. El contenido total de polifenoles en el extracto hidroalcohólico de maca roja fue de $1,60 \mathrm{mg}$ pyrogallol/100 $\mathrm{mg}$ de extracto hidroalcohólico de maca roja.

\section{PROTOCOLO EXPERIMENTAL}

Para el presente estudio, las ratas fueron divididas al azar en los siguientes seis grupos: Grupo 1 (PO): ratas $\mathrm{PO}$ las cuales recibieron agua destilada; Grupo 2 (OVX): ratas ovariectomizadas tratadas con agua destilada; Grupo $3\left(\mathrm{OVX}+\mathrm{E}_{2}\right)$ : ratas $\mathrm{OVX}$ tratadas con estradiol; Grupo 4-6 (OVX+MR1, OVX+MR2 y OVX+MR3): ratas OVX tratadas con tres diferentes dosis de maca roja. Estudios previos han mostrado que el extracto hidroalcohólico de maca roja con un contenido de 4,3 $\mathrm{mg}$ polifenoles/kg de peso corporal fue capaz de proteger la estructura ósea de ratas ovariectomizadas (20) y, por esta razón, las ratas OVX de los grupos 4, 5 y 6 recibieron extractos hidroalcohólicos de maca roja las cuales contuvieron 2,15, 4,3 y 8,6 mg polifenoles/kg de peso corporal, respectivamente. El agua destilada, el estradiol (4 ug/kg) y la maca roja fueron administrados por vía oral $(1 \mathrm{~mL})$ durante 28 días usando una sonda nasogástrica N. ${ }^{\circ} 18$ (Fisher Scientific, Pittsburgh, Pennsylvania).

Al final del experimento las ratas fueron sacrificadas, la 
sangre fue colectada del tronco cervical y el suero fue separado por centrifugación a $1000 \mathrm{~g}$ para los ensayos de determinación del IFN-g. Después de eso, el útero fue disecado, separado del tejido conectivo circundante y pesado. El peso corporal también fue registrado de forma semanal desde el inicio del experimento.

\section{DETERMINACIÓN DE IFN-g}

Las concentraciones de IFN-g en suero fueron determinadas usando un kit de ELISA para IFN-g de rata proveniente de BD Bioscience (CA, USA; catálogo: 558861). Las mediciones fueron realizadas por espectrofotometría a $570 \mathrm{~nm}$. Todas las muestras fueron corridas en el mismo tiempo para evitar variaciones inter-ensayo. Los datos se expresan en $\mathrm{pg} / \mathrm{mL}$.

\section{ANÁLISIS ESTADÍSTICOS}

Los datos fueron analizados usando el paquete estadístico Stata versión 8,0 (Stata Corporation, College Station, TX, USA). Los datos son presentados como la media \pm desviación estándar (DE). Las diferencias entre los grupos fue determinada usando el análisis de varianza (ANOVA) y la prueba post-hoc Scheffé para las variables que presentaron distribución normal (peso corporal y uterino) o la prueba de Mann-Whitney $U$ para variables sin distribución normal (niveles de IFN-g). El peso uterino relativo se calculó según el índice: (peso uterino/ peso corporal final) $\times 100$. Los valores $p<0,05$ fueron considerados como estadísticamente significativas.

\section{CONSIDERACIONES ÉTICAS}

Todos los procedimientos con animales fueron realizados siguiendo las normas de la Guía para el

Tabla 1. Efecto de la maca roja (Lepidium meyenii) en el peso corporal final y el peso uterino seco relativo en ratas ovariectomizadas

\begin{tabular}{lccc}
\hline & $\begin{array}{c}\text { Peso corporal } \\
\text { inicial }(\mathrm{g})\end{array}$ & $\begin{array}{c}\text { Peso corporal } \\
\text { final }(\mathrm{g})\end{array}$ & $\begin{array}{c}\text { Peso relativo } \\
\text { uterino seco }\end{array}$ \\
\hline PO & $223,67 \pm 2,29$ & $244,50 \pm 2,83$ & $0,95 \pm 0,31$ \\
OVX & $249,67 \pm 3,93$ & $287,83 \pm 4,76$ & $0,36 \pm 0,11$ \\
OVX + E & $232,67 \pm 5,01$ & $256,17 \pm 5,75$ & $0,77 \pm 0,17$ \\
OVX + MR1 & $177,33 \pm 5,97$ & $196,83 \pm 7,46$ & $0,24 \pm 0,08$ \\
OVX + MR2 & $195,00 \pm 4,65$ & $213,67 \pm 4,58$ & $0,18 \pm 0,06$ \\
OVX + MR3 & $210,17 \pm 6,47$ & $233,83 \pm 4,25$ & $0,20 \pm 0,04$ \\
\hline
\end{tabular}

Los datos están expresados como la media \pm desviación estándar PO: pseudoovariectomizadas; OVX: ovariectomizadas; OVX+E2: ratas ovariectomizadas tratadas con estradiol; OVX+MR1: ratas ovariectomizas tratadas con $\mathrm{XX}$ de maca roja; OVX+MR2: ratas ovariectomizas tratadas con $\mathrm{XX}$ de maca roja; OVX+MR3: ratas ovariectomizadas tratadas con $X X$ de maca roja

${ }^{a} p<0,05$ vs. ratas OVX; ${ }^{b} p<0,05$ vs. Grupo PO; ${ }^{c} p<0,05$ vs. Grupo OVX $+\mathrm{E} 2$ cuidado y uso de animales de laboratorio ${ }^{(26)}$. El Comité de Ética Institucional de la Oficina de Investigación Científica de la Universidad Peruana Cayetano Heredia aprobó previamente el estudio (SIDISI-UPCH: 57053, 13 de agosto de 2010).

\section{RESULTADOS}

Al final del experimento, las ratas OVX mostraron un incremento en el peso corporal cuando fueron comparadas con las ratas de los grupos $\mathrm{PO}, \mathrm{OVX}+\mathrm{E}_{2}$, y las tratadas con las diferentes dosis de maca roja $(p<0,05)$. No se encontraron diferencias significativas entre las ratas $\mathrm{PO}$ y las tratadas con $\mathrm{E}_{2}$ y diferentes dosis de maca roja $(p>0,05)$. Los pesos corporales finales fueron similares entre los grupos OVX y los grupos tratados con $\mathrm{E}_{2}$ y maca roja $(p>0,05)$ (Tabla 1).

Las ratas OVX mostraron una reducción del peso uterino seco relativo cuando se les comparó con las ratas $\mathrm{PO}$, y las tratadas con $E_{2}$ y maca roja $(p<0,05)$. Se reportó un mayor peso uterino seco relativo en ratas OVX que fueron tratadas con E2 en comparación con aquellas ratas OVX tratadas con tres diferentes dosis de maca roja $(p<0,05)$. No hubo diferencias significativas entre las ratas OVX con aquellas tratadas con maca $(p>0,05)$ (Tabla 1). La Figura 1 muestra el efecto del tratamiento con tres diferentes dosis de maca roja sobre los niveles séricos de IFN-g en ratas OVX.

La prueba no paramétrica Mann-Whitney U mostró menores niveles de IFN-g en ratas OVX en comparación con las ratas $\mathrm{PO}(155 \pm 21,16$ vs. $703,94 \pm 161,79 \mathrm{pg} / \mathrm{mL} ; p<0,05)$. El tratamiento con estradiol $(1784,46 \pm 472,58 \mathrm{pg} / \mathrm{mL})$

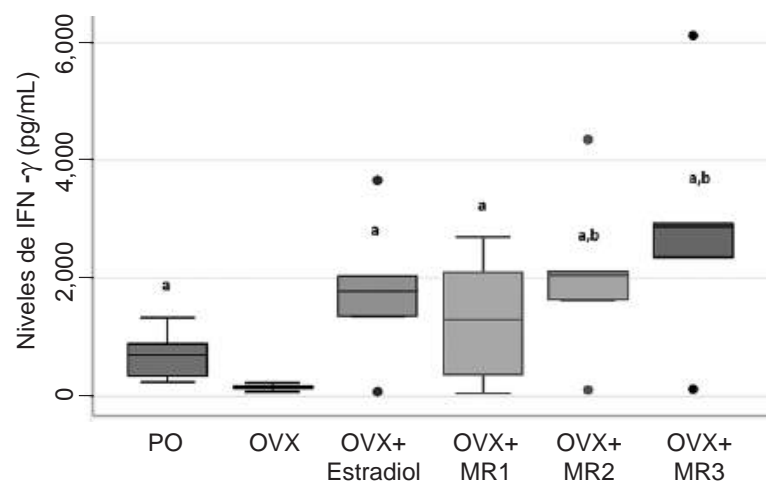

Figura 1. Efecto de la maca roja sobre los niveles de IFN-g en ratas ovariectomizadas

Los datos están expresados como la media $\pm \mathrm{DE}$

PO: pseudoovariectomizadas; OVX: ovariectomizadas; OVX+E2: ratas ovariectomizadas tratadas con estradiol; OVX+MR1: ratas ovariectomizadas tratadas con $X X$ de maca roja; OVX+MR2: ratas ovariectomizadas tratadas con $X X$ de maca roja; OVX+MR3: ratas ovariectomizadas tratadas con $\mathrm{XX}$ de maca roja 
revirtió el efecto de la ovariectomía, mostrando que el peso uterino fue mayor que los observados en los grupos OVX y $\mathrm{PO}(p<0,05)$. Las ratas OVX tratadas con la menor dosis de maca roja presentaron mayores niveles de IFN-g que las ratas OVX (OVX+MR1: $1306,80 \pm 409,56 \mathrm{pg} / \mathrm{mL} ; p<0,05)$; mientras tanto, las ratas OVX tratadas con las dosis mayores de maca roja (OVX+MR2: 2051,64 \pm 556,07 pg/mL, y OVX+MR3: $2881,09 \pm 782,51 \mathrm{pg} / \mathrm{mL}$ ) reportaron mayores valores de IFN-g que aquellas ratas de los grupos OVX y PO $(p<0,05)$. La maca roja mostró un incremento dosisrespuesta en los niveles de IFN-g $(r=0,57, p<0,05)$.

\section{DISCUSIÓN}

La ovariectomía ha sido ampliamente usada como un modelo in vivo para imitar los síntomas posmenopaúsicos tales como la pérdida ósea ${ }^{(27)}$. Se apreció el efecto de la ovariectomía con la disminución del peso uterino como fue notado por otros estudios (27). Además, la ovariectomía causo una reducción en los niveles de IFN-g, lo cual también se relaciona a la deficiencia de estrógeno observado en animales ovariectomizados ${ }^{(3)}$.

Se sabe que niveles bajos de estrógenos pueden producir un desbalance del radio entre Th1 y $\mathrm{TH} 2$ (28) (radio Th1/Th2). Los linfocitos Th1 producen principalmente citoquinas proinflamatorias tales como IFN-g, IL-2. TNFa y otras, favoreciendo la inmunidad celular relacionada a la inmunidad innata ${ }^{(29)}$. Además, se ha demostrado que la administración exógena de estradiol en la THR incrementa los niveles de Th1 ${ }^{(3)}$. Los resultados del presente estudio muestran que el estradiol incrementa los niveles de IFN-g en ratas ovariectomizadas los cuales, a su vez, puede resultar en un aumento de los valores de Th1 ${ }^{(3)}$.

Además, se ha observado que las tres diferentes dosis de maca roja administradas $(2,15,4,3$ y $8,6 \mathrm{mg}$ polifenoles $/ \mathrm{kg}$ ) pueden incrementar los niveles de IFN-g en una manera dosis dependiente favoreciendo la inmunidad innata. Previamente, se ha observado que la maca roja es capaz de restaurar la arquitectura ósea (20) y de tener actividad antidepresiva ${ }^{\left({ }^{(0)}\right.}$ en animales OVX.

El hecho de que estos efectos de la maca roja no están relacionados con el incremento del peso uterino como se observan en otros estudios ${ }^{(20,31)}$ apoya el hecho de que la maca roja no presenta un efecto similar a estrógenos ${ }^{(20,31)}$. También se ha sugerido que la maca roja podría actuar como un modulador selectivo de los receptores de estrógenos (SERM) ${ }^{(20)}$. El incremento de los niveles de IFN-g ayuda al organismo a la defensa contra patógenos intracelulares, por la activación de macrófagos. De hecho, un estudio previo ha demostrado el efecto de la maca contra los virus de influenza humana tipo A y B corroborando los resultados de este estudio y sugiriendo el efecto antiviral de la maca ${ }^{(32)}$. Futuros estudios son necesarios para determinar los efectos del extracto maca roja y sus fracciones sobre los niveles de marcadores para Th1 y Th2 y su efecto sobre el sistema inmune y su posible uso alternativo para tratamientos contra diversas enfermedades.

La maca roja presenta el mayor contenido de polifenoles ${ }^{(20)}$. Por esta razón, el presente estudio utilizó un extracto hidroalcohólico de maca roja estandarizado. Además, se ha probado que los polifenoles pueden ser potenciales antioxidantes ${ }^{(33,34)}$. Se sabe que la maca contiene flavonas, quercetina y antocianinas (24) las cuales tienen actividad antioxidante ${ }^{(33,34)}$. Estudios previos han demostrado que los polifenoles pueden incrementar los niveles de IFN-g (35) y que algunos flavonoides pueden mejorar la resistencia epitelial (35) la cual también está relacionada con la inmunidad innata.

En conclusión, la maca roja puede incrementar los niveles de IFN-g en ratas ovariectomizadas de forma dosis-respuesta, un modelo animal que induce síntomas relacionados a la posmenopausia. De esta forma, la maca roja puede considerarse un tratamiento alternativo para enfermedades relacionadas con la edad y a la deficiencia de estrógenos como la osteoporosis.

Agradecimientos: Los autores agradecen el trabajo de Isaías Cárdenas para el presente estudio. Además, dan especiales gracias al apoyo financiero de la Facultad de Medicina de la Universidad Peruana Cayetano Heredia. A Ing. Alfonso higa de Agroindustrial Chanchamayo (Lima, Perú). A Irma Fernández, botánica del Departamento de Ciencias Farmacéuticas de la Universidad Peruana Cayetano Heredia.

Contribuciones de autoría: JLR, FGC y GFG participaron en la concepción y diseño del protocolo del estudio y en la obtención del financiamiento. JLR, FGC, POM, JR y CG participaron en la recolección y obtención de resultados; además contribuyeron en el diseño del artículo. JR participó en la realización del análisis estadístico de los datos. JLR, GFG y JR participaron en el análisis y redacción del artículo. Todos los autores han dado su revisión crítica del artículo.

Fuente de financiamiento: Facultad de Medicina, Universidad Peruana Cayetano Heredia.

Conflictos de interés: GFG es gerente general de Cayenatur EIRL, empresa dedicada a productos naturales. Los otros autores declaran no tener conflicto de interés para este artículo. 


\section{REFERENCIAS BIBLIOGRÁFICAS}

1. Appt SE, Clarkson TB, Hoyer PB, Kock ND, Goode AK, May MC, et al. Experimental induction of reduced ovarian reserve in a nonhuman primate model (Macaca fascicularis). Comp Med. 2010 Oct;60(5):380-8.

2. Sacco SM, Ward WE. Revisiting estrogen: efficacy and safety for postmenopausal bone health. J Osteoporos. 2010:708931.

3. Kumru S, Godekmerdan A, Yilmaz B. Immune effects of surgical menopause and estrogen replacement therapy in peri-menopausal women. J Reprod Immunol. 2004 Aug;63(1):31-8.

4. De Martinis M, Di Benedetto MC, Mengoli LP, Ginaldi L. Senile osteoporosis: is it an immunemediated disease? Inflamm Res. 2006 Oct;55(10):399-404.

5. Breuil V, Ticchioni M, Testa J, Roux $\mathrm{CH}$, Ferrari P, Breittmayer JP, et al. Immune changes in post-menopausal osteoporosis: the Immunos study. Osteoporos Int. 2010 May;21(5):805-14. doi: 10.1007/ s00198-009-1018-7.

6. Stopińska-Głuszak U, Waligóra J, Grzela T, Głuszak M, Jóźwiak J, Radomski D, et al. Effect of estrogen/ progesterone hormone replacement therapy on natural killer cell cytotoxicity and immunoregulatory cytokine release by peripheral blood mononuclear cells of postmenopausal women. J Reprod Immunol. 2006 Feb;69(1):65-75.

7. Porter VR, Greendale GA, Schocken $\mathrm{M}$, Zhu X, Effros RB. Immune effects of hormone replacement therapy in post-menopausal women. Exp Gerontol. 2001 Feb;36(2):311-26.

8. Yang JH, Chen CD, Wu MY, Chao $\mathrm{KH}$, Yang YS, Ho HN. Hormone replacement therapy reverses the decrease in natural killer cytotoxicity but does not reverse the decreases in the T-cell subpopulation or interferon-gamma production in postmenopausal women. Fertil Steril. 2000 Aug;74(2):261-7.

9. Pinkerton JV, Stovall DW, Kightlinger RS. Advances in the treatment of menopausal symptoms. Womens Health (Lond Engl). 2009 Jul;5(4):361-384; quiz 383-4. doi: 10.2217/whe.09.31.
10. Weitzmann MN, Pacifici R. Estrogen regulation of immune cell bone interactions. Ann N Y Acad Sci. 2006 Apr;1068:256-74.

11. Moskowitz D. A comprehensive review of the safety and efficacy of bioidentical hormones for the management of menopause and related health risks. Altern Med Rev. 2006 Sep;11(3):208-23.

12. Gonzales GF, Ruiz A, Gonzales C, Villegas L, Cordova A. Effect of Lepidium meyenii (maca) roots on spermatogenesis of male rats. Asian J Androl. 2001 Sep;3(3):231-3.

13. Gonzales GF1, Gasco M, Córdova A, Chung A, Rubio J, Villegas L. Effect of Lepidium meyenii (Maca) on spermatogenesis in male rats acutely exposed to high altitude $(4340 \mathrm{~m})$. J Endocrinol. 2004 Jan;180(1):87-95.

14. Rubio J, Riqueros MI, Gasco M, Yucra S, Miranda S, Gonzales GF. Lepidium meyenii (Maca) reversed the lead acetate induced -- damage on reproductive function in male rats. Rubio J1, Riqueros MI, Gasco M, Yucra S, Miranda S, Gonzales GF.

15. Cicero AF, Bandieri E, Arletti R. Lepidium meyenii Walp. improves sexual behaviour in male rats independently from its action on spontaneous locomotor activity. J Ethnopharmacol. 2001 May;75(23):225-9.

16. Cicero AF1, Piacente S, Plaza A, Sala E, Arletti R, Pizza C. Hexanic Maca extract improves rat sexual performance more effectively than methanolic and chloroformic Maca extracts. Andrologia. 2002 Jun;34(3):177-9.

17. Ruiz-Luna AC, Salazar S, Aspajo NJ, Rubio J, Gasco M, Gonzales GF. Lepidium meyenii (Maca) increases litter size in normal adult female mice. Reprod Biol Endocrinol. 2005;3:16.

18. Gonzales GF, Miranda S, Nieto J, Fernández G, Yucra S, Rubio J, et al. Red maca (Lepidium meyenii) reduced prostate size in rats. Reprod Biol Endocrinol 2005;3:5.

19. Gonzales C, Rubio J, Gasco M, Nieto J, Yucra S, Gonzales GF. Effect of short-term and long-term treatments with three ecotypes of Lepidium meyenii (MACA) on spermatogenesis in rats. J Ethnopharmacol. 2006 Feb 20;103(3):448-54.
20. Gonzales C, Cárdenas-Valencia I, Leiva-Revilla J, Anza-Ramirez C, Rubio J, Gonzales GF. Effect of different varieties of Maca (Lepidium meyenii) on bone structure in ovairectomized rats. Forsch Komplementmed. 2010;17(3):137-43. doi: 10.1159/000315214.

21. Zhang Y, Lai WP, Leung PC, Wu CF, Wong MS. Short- to mid-term effects of ovariectomy on bone turnover, bone mass and bone strength in rats. Biol Pharm Bull. 2007 May;30(5):898-903.

22. Alzamora L, Galván P, Alvarez E, Torres D, Colona E, Aliaga M, et al. Producción de IFN- $\gamma$ en cultivos de linfocitos humanos por efecto de los extractos metabólicos de cuatro ecotipos de Lepidium meyenii, Chacón (Brassicaceae). Rev Peru Biol. 2007(Jul);13(3):207-9.

23. RubioJ, Qiong W, LiuX, JiangZ, DangH, Chen SL, et al. Aqueous extract of black maca (Lepidium meyenii) on memory impairment induced by ovariectomy in mice. Evid Based Complement Alternat Med. 2011;2011:253958. doi: 10.1093/ ecam/nen063.

24. Valerio LG Jr, Gonzales GF. Toxicological aspects of the South American herbs cat's claw (Uncaria tomentosa) and Maca (Lepidium meyenii): a critical synopsis. Toxicol Rev. 2005;24(1):11-35.

25. Kähkönen MP, Hopia AI, Vuorela HJ, Rauha JP, Pihlaja K, Kujala TS, et al. Antioxidant activity of plant extracts containing phenolic compounds. J Agric Food Chem. 1999 Oct;47(10):3954-62.

26. The National Academy of Sciences. Guide for the Care and Use of Laboratory Animals. 8th ed. Washington, D.C.; The National Academies Press: 1995.

27. Gallo D, Battaglia A, Mantuano E, Travaglia D, De Stefano I, Buzzonetti A, et al. 17beta-Estradiol and soy phytochemicals selectively induce a type 2 polarization in mesenteric lymph nodes of ovariectomized rats. Menopause. 2008 Jul-Aug;15(4 Pt 1):718-25. doi: 10.1097/ gme.0b013e318167b92a.

28. Salem ML. Estrogen, a double-edged sword: modulation of TH1 - and TH2mediated inflammations by differential 
regulation of $\mathrm{TH} 1 / \mathrm{TH} 2$ cytokine production. Curr Drug Targets Inflamm Allergy. 2004 Mar;3(1):97-104.

29. Mosmann TR, Sad S. The expanding universe of T cell subsets: Th1, Th2 and more. Immunol Today. 1996 Mar;17(3):138-46.

30. Rubio J, Caldas M, Dávila S, Gasco M, Gonzales GF. Effect of three different cultivars of Lepidium meyenii (Maca) on learning and depression in ovariectomized mice. BMC Complement Altern Med. 2006;6:23.

31. Scalbert A, Manach C, Morand C, Rémésy C, Jiménez L. Dietary polyphenols and the prevention of diseases. Crit Rev Food Sci Nutr. 2005;45(4):287-306.

32. Del Valle Mendoza J, Pumarola T, Gonzales LA, Del Valle LJ. Antiviral activity of maca (Lepidium meyenii) against human influenza virus. Asian Pac J Trop Med. 2014 Sep;7S1:S415-20. doi: 10.1016/S1995-7645(14)60268-6.

33. Weaver CM, Barnes S, Wyss JM, Kim H, Morré DM, Morré DJ, et al. Research Highlights from the PurdueUAB Botanicals Research Center for Age Related Diseases. Pharm Biol. 2009 Aug 1;47(8):768-773.

34. Miles EA, Zoubouli P, Calder PC. Effects of polyphenols on human Th1 and Th2 cytokine production. Clin Nutr. 2005 Oct;24(5):780-4.

35. Magrone T, Candore G, Caruso C, Jirillo E, Covelli V. Polyphenols from red wine modulate immune responsiveness: biological and clinical significance. Curr Pharm Des. 2008;14(26):2733-48.

Correspondencia: Julio Rubio Dirección: Av. Honorio Delgado s/n, Urb. Ingeniería. San Martín de Porres

Teléfono: (+511) 3190000 anexo 2515

Correoelectrónico:julio.rubio.m@upch.pe

\section{Visite nuestra página en Facebook, www.facebook.com/rpmesp Infórmese sobre los eventos y los nuevos contenidos de la Revista Peruana de Medicina Experimental y Salud Pública}

\section{REVISTA PERUANA DE MEDICINA EXPERIMENTAL Y SALUD PÚBLICA} Difundiendo investigación en salud

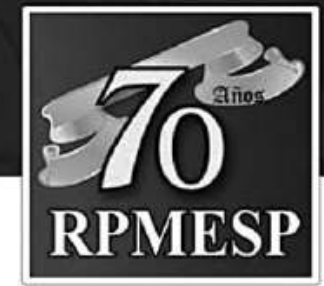
1942 - 2012

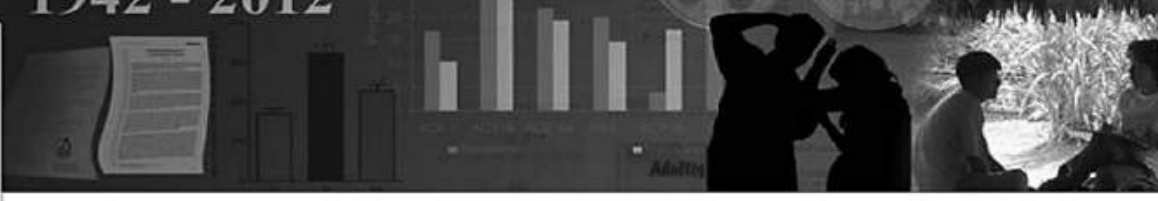

Revista Peruana de Medicina Experimental y

Salud Pública (OFICIAL)

A 1254 personas les gusta esta página · 8 personas están hablando de esto

Comunidad

La Revista Peruana de Medicina Experimental y Salud Publica es el órgano oficial de difusión cientifica del Instituto Nacional de Salud

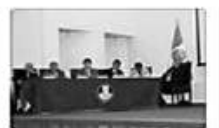

Fotos
1254

SIMPOSIO SAR DE

SIMPOSIO SALUD El simposio de verdar estuvo muy bueno. $M$ Notas 5 